Article

\title{
A Naked Eye Refractive Index Sensor with a Visible Multiple Peak Metamaterial Absorber
}

\author{
Heli Ma, Kun Song, Liang Zhou and Xiaopeng Zhao * \\ Smart Materials Laboratory, Department of Applied Physics, Northwestern Polytechnical University, \\ Xi'an 710129, China; E-Mails: marcos12@126.com (H.M.); songkun@mail.nwpu.edu.cn (K.S.); \\ mzlmql@126.com (L.Z.)
}

* Author to whom correspondence should be addressed; E-Mail: xpzhao@nwpu.edu.cn; Tel.: +86-29-8843-1662.

Academic Editor: Omar M. Ramahi

Received: 18 November 2014 / Accepted: 19 March 2015 / Published: 26 March 2015

\begin{abstract}
We report a naked eye refractive index sensor with a visible metamaterial absorber. The visible metamaterial absorber consisting of a silver dendritic/dielectric/metal structure shows multiple absorption peaks. By incorporating a gain material (rhodamine B) into the dielectric layer, the maximal magnitude of the absorption peak can be improved by about $30 \%$. As the metamaterial absorber is sensitive to the refractive index of glucose solutions, it can function as a sensor that quickly responds to variations of the refractive index of the liquid. Meanwhile, since the response is presented via color changes, it can be clearly observed by the naked eyes. Further experiments have confirmed that the sensor can be used repeatedly.
\end{abstract}

Keywords: refractive index sensor; naked eyes; metamaterial absorber; bottom-up

\section{Introduction}

Metamaterials (MMs) are artificial media exhibiting many extraordinary electromagnetic (EM) properties that do not exist in the naturally available materials, for instance, negative refractive index, super-resolution and trapped rainbow effects [1-3]. With these unique properties, MMs may lead to important practical applications [4-8]. As an important branch of MMs, metamaterial absorbers (MAs) are of great current interest due to their intriguing properties including high absorption of EM energy and 
hyperspectral imaging [9]. In order to maximize the absorption of EM waves, meticulously designed metal resonator/dielectric/metal composite structures are used to control the effective permittivity and permeability of a MA. By optimizing the geometric parameters, the MA can neither reflect nor transmit EM waves at some selective frequency band. The EM energy is therefore weakened to zero with a high absorption coefficient. In the past few years, research on MAs has undergone an almost explosive growth, and considerable progress has been made in this burgeoning field. The current interest in the field is turning to the enticingly potential applications in areas such as sensors, thermal emitters, and photovoltaic devices $[10,11]$. However, to date the reported previously MAs could only realize near unity absorbance from the microwave to the infrared region [12-17]. Thus, how to realize a MA operating in visible light region is still a challenge for researchers.

Recently, much attention has been focused on optimizing MM properties with gain materials [18-20]. Xiao et al. [18] incorporated a gain material in the high-local-field area of a fishnet MM to improve the loss compensation. This idea provides a flexible route to control the properties of MMs. Fang et al. [20] investigated the optical response of a realistic split-ring resonator (SRR) array with a gain layer underneath. Results showed that the SRR resonance was enhanced and the real and imaginary parts of permeability $\mu$ became larger than those without gain layers. From these works we can see that the relation between the enhancement of resonance and absorption property of MA deserves careful research.

In this article, we experimentally demonstrate a MA consisting of silver dendritic cells with different diameters that exhibits multiband absorption peaks in the visible light region. By incorporating moderate amounts of rhodamine $\mathrm{B}$ ( $\mathrm{RhB}, 99 \%$ pure, laser grade) into the dielectric layer, the absorption magnitudes of the MA can be effectively enhanced. Interestingly, the MA can quickly respond to the variations of the concentrations of glucose solutions to yield color changes on the surface of the MA observable by the naked eyes, enabling important application as a medical sensor.

\section{Experimental}

The fabrication process of this multiband visible MA obtained by a "bottom-up" electrochemical deposition method is shown in Figure 1a. The preparation of the silver dendritic cells has been reported in detail [21]. We used ITO conductive glass as cathode, a planar and smooth high-purity silver slice as anode. A mixed solution of silver nitrate $\left(\mathrm{AgNO}_{3}\right) /$ polyethylene glycol-20000 (PEG-20000, concentration $9.9 \% \sim 10.7 \%$ ) was injected into the interspace of the two electrodes. Under the condition of direct current (DC) voltages of $0.8 \sim 0.9 \mathrm{~V}$ for $80 \sim 100 \mathrm{~s}$ at room temperature, silver dendritic cells with different diameters ranging from $70 \mathrm{~nm}$ to $280 \mathrm{~nm}$ were fabricated, as shown in Figure $1 \mathrm{~b}$. The preparation of the silver metal plane used the same electrochemical deposition device. Firstly, $0.1 \mathrm{~g}$ of $\mathrm{AgNO}_{3}$ was added into $2 \mathrm{~mL}$ ultrapure water (UPW) and stirred sufficiently. Then, $1 \mathrm{~mL}$ triethanolamine (TEA) was added into the solution drop by drop. Immediately, a brown precipitate formed in the mixture, however, as the amount of added TEA increased further, the precipitate gradually disappeared and the solution became clear. Essentially, the silver ions were complexed with TEA molecules. With the solution of $\mathrm{AgNO}_{3} /$ triethanolamine (TEA) mixture a silver metal plane with a thickness of about $400 \mathrm{~nm}$ was prepared, as shown in Figure 1c. In the experimental procedure, the deposition current decreased with increasing deposition time. The ITO conductive glass coated with silver dendritic cells and silver metal plane took on silver-grey and silver-white colors, respectively. A 
polyvinyl alcohol (PVA) solution with concentration of 1\% 3\% was dropped onto the surface of the prepared silver dendritic cells. By this vertical deposition method under ambient conditions, the silver dendritic cells were covered with a PVA dielectric interlayer with a thickness of about 50 100 nm [21]. Finally, the silver dendritic cells/PVA dielectric interlayer and silver metal plane were combined face-to-face to form the MA sample. The sample area finallyobtained is about $30 \mathrm{~mm} \times 12 \mathrm{~mm}$.
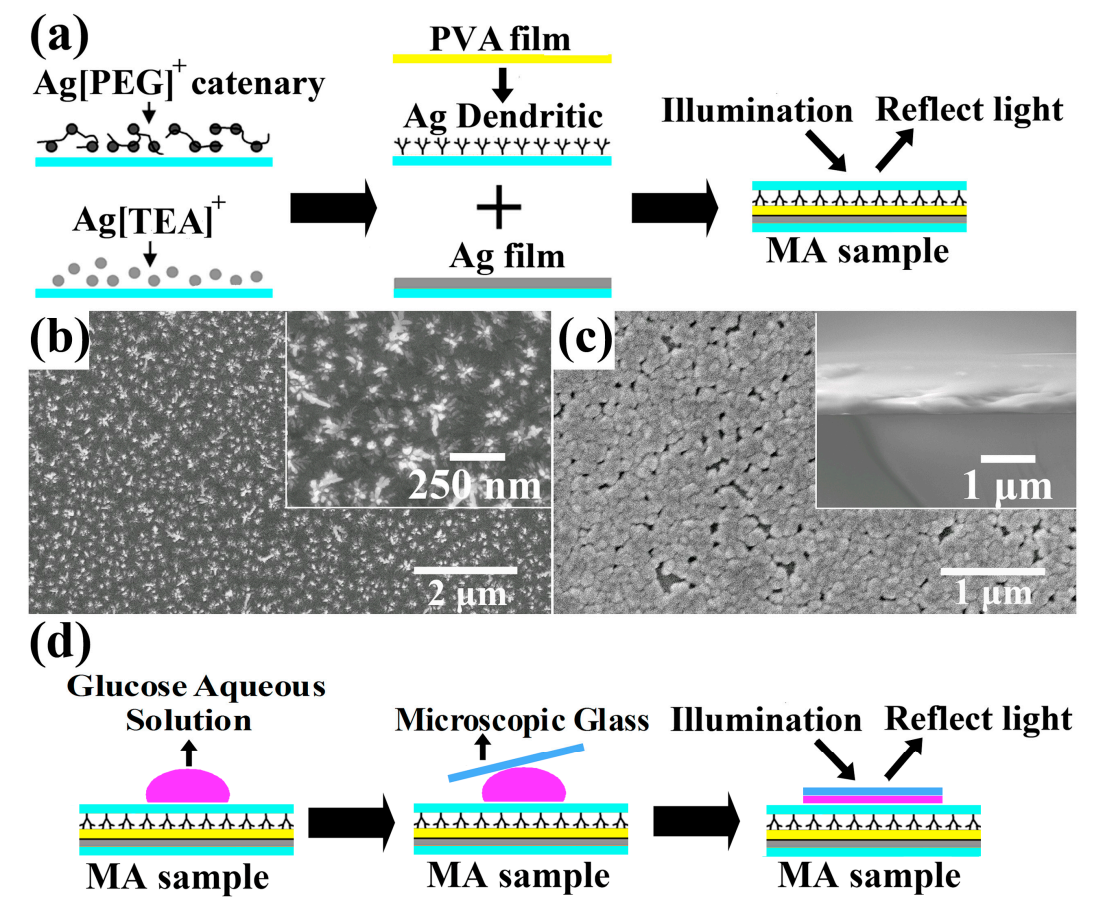

Figure 1. (a) The fabrication process of the multiband visible MA; (b) Scanning electron microscope (SEM) image of the prepared silver dendritic units - the illustration is an enlarged drawing of silver dendritic units; (c) SEM image of the silver metal plane - the inset is the cross-section of the silver metal plane.

In practical terms, two kinds of MA samples were prepared. The silver dendritic cells of the first kind of MA samples were obtained under a deposition voltage of $0.8 \mathrm{~V}$, a deposition time of $80 \mathrm{~s}$, a PEG concentration of $10.7 \%$ and $1.0 \%$ PVA. The silver dendritic cells of the second kind of MA samples were prepared with a deposition voltage of $0.8 \mathrm{~V}$, a deposition time of $100 \mathrm{~s}$, a $9.9 \%$ concentration of PEG and $3.0 \%$ PVA. In addition, to investigate the effect of gain material $(\mathrm{RhB})$ on the absorption properties of the MA samples, we incorporated RhB into the PVA dielectric layer instead of a pure PVA layer.

\section{Results and Discussion}

\subsection{Measurements of Absorption Properties of MA Samples}

The MA samples were measured via a UV-visible spectrophotometer (Hitachi UV-4100) in reflection mode in the wavelength region of $500 \sim 800 \mathrm{~nm}$. The incident and reflection angles were both $5^{\circ}$, and the reflection spectra were normalized to that of a silver mirror. Since the silver metal plane is dense and its thickness is considerably larger than the penetration depth of light wave, the transmittance can be 
ignored. As a result, the absorptivity can be calculated as $\mathbf{A}=1-\mathbf{R}$, where $\mathbf{A}$ and $\mathbf{R}$ refer to the absorptivity and reflectivity of the sample, respectively.

Zhu et al. [22,23] have investigated the absorption performances of the MAs with periodically and randomly arrayed silver dendritic cells via simulations. The results indicated that the power loss might be attributed to the electric resonance caused by the main trunk of dendritic cells and magnetic resonance induced by the antisymmetrical current between the dendritic cells and metal plane. Figure 2 a shows the measured absorption spectra of the proposed MA, MA integrated with RhB, and ITO glass coated with PVA. The MA samples were prepared under the first kind of deposition conditions. It is seen that the present MA with different silver dendritic cells can simultaneously resonate at different wavelengths leading to multiband absorption. However, the quantity and density of the silver dendritic cells with the similar geometric parameters are so few that it is unfavorable for the generation of strong resonance at a certain frequency. Consequently, a unit absorptivity cannot be realized and the maximum absorptivity of the MA is about 0.537 at $524 \mathrm{~nm}$. After incorporating RhB into the PVA dielectric layer, it is of significance that the magnitudes of the absorption peaks of the proposed MA are effectively enhanced. The maximal absorptivity is dynamically increased by about $30 \%$. This result arises due to the inherent absorption of the $\mathrm{RhB}$ which results in a significant decrease of the reflectance (Figure $2 b$ ). Meanwhile, compared with the sample without $\mathrm{RhB}$, the absorption peaks of the sample integrated with $\mathrm{RhB}$ show significant red shifts. These spectral shifts are attributed to the fact that the maximum absorption peak of $\mathrm{RhB}$ is at $555 \mathrm{~nm}$ wavelength which is in the long-wavelength part of the absorption peak for the MA sample without $\mathrm{RhB}$, so after the inclusion of $\mathrm{RhB}$, the absorption peaks were red shifted [24]. Furthermore, there remains a weak absorption of about 0.14 to 0.3 owing to the inherent absorption of the ITO glass and PVA.
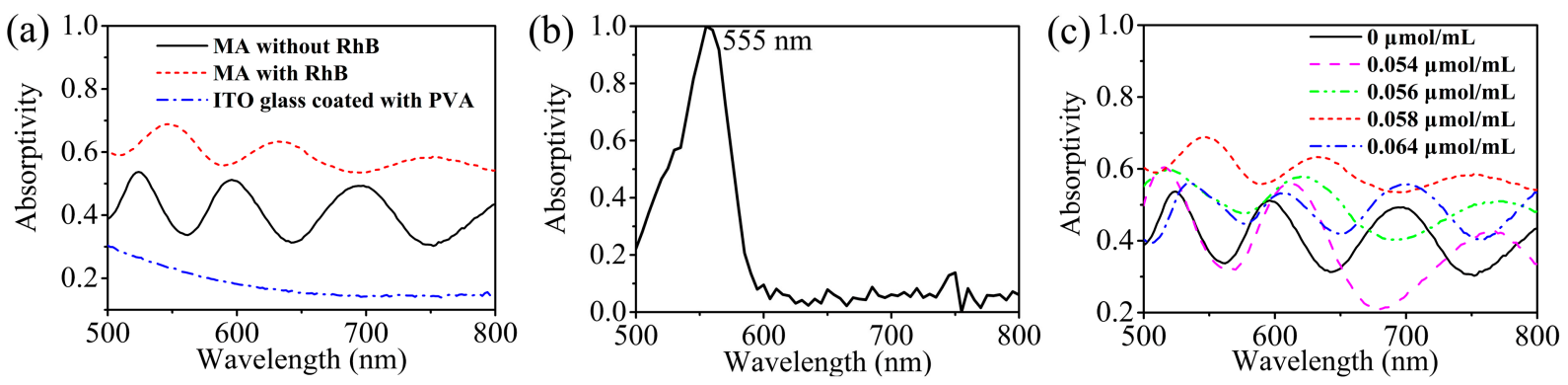

Figure 2. (a) The absorption spectra for MA without RhB, MA with RhB, and the ITO glass coated with PVA in the testing region of 500 800 nm; (b) The inherent absorption spectrum of the RhB; (c) The absorption spectra of the MA evolve with different concentrations of RhB.

Figure 2c illustrates the evolution of the absorption spectra of the MAs with different concentrations of RhB. The magnitudes and locations of the absorption peaks measured under different concentrations of $\mathrm{RhB}$ are shown in Table 1 . It is obvious that in response to the increasing concentration of $\mathrm{RhB}$, the magnitudes of the absorption peaks gradually increase and rise to their maximal values $(0.692 / 546 \mathrm{~nm}$, $0.641 / 632 \mathrm{~nm}, 0.596 / 752 \mathrm{~nm}$ ) under the concentration of $0.058 \mu \mathrm{mol} / \mathrm{mL}$. However, with the further increase of the concentration of $\mathrm{RhB}$, the magnitudes of the absorption peaks gradually decrease. When the concentration of $\mathrm{RhB}$ is $0.064 \mu \mathrm{mol} / \mathrm{mL}$, the average absorption decreases significantly. This phenomenon may be explained by the fact that the $\mathrm{RhB}$ will generate photoluminescence under a direct 
light illumination, effectively increasing the reflection of the MA sample, which leads to a significant decrease of the final absorption of the MA sample [24].

Table 1. The magnitudes and locations of absorption peaks under different concentrations of RhB.

\begin{tabular}{cccc}
\hline Concentration $(\boldsymbol{\mu m o l} / \mathbf{m L})$ & \multicolumn{3}{c}{ The Magnitudes/Locations of Absorption Peaks $(\mathbf{n m})$} \\
\hline 0 & $0.537 / 524$ & $0.513 / 596$ & $0.502 / 698$ \\
0.054 & $0.608 / 516$ & $0.564 / 612$ & $0.430 / 768$ \\
0.056 & $0.600 / 518$ & $0.584 / 620$ & $0.516 / 770$ \\
0.058 & $0.692 / 546$ & $0.641 / 632$ & $0.596 / 752$ \\
0.064 & $0.560 / 536$ & $0.538 / 606$ & $0.506 / 700$ \\
\hline
\end{tabular}

\subsection{Measurements of the Sensibility of MA to Variations of the Refractive Index for Glucose} Aqueous Solutions

To further investigate the intriguing properties of the present MA, we measured the sensibility of MA to the variations of the refractive index of aqueous glucose (GLU) solutions of different concentrations. Generally, refractive index sensors based on photonic crystals which need to inject the tested liquid into the microcavity can be used only once or require a complex process for reuse, extremely limiting their practical applications $[25,26]$. In our experiments, the GLU aqueous solution drop was overlaid on the surface of ITO glass deposited with silver dendritic cells and pressed into a thin film by a microscopic glass. The fabrication and measurement processes are shown in Figure 1d. In this manner, the PVA layer does not dissolve in the aqueous solution and the sample can be repeatedly used just by removing the GLU aqueous solution on its surface. The MA sample was obtained via the second deposition conditions. We tested five kinds of concentrations for $\operatorname{GLU}(0,10,15,25$, and $60 \%)$ with corresponding refractive indexes of about 1.333, 1.348, 1.356, 1.375, and 1.439 at $589 \mathrm{~nm}$, respectively [10,27]. The refractive index change range is only 0.106. As shown in Figure 3, there are four absorption peaks at $514,562,630$ and $732 \mathrm{~nm}$ before any solution is dropped.

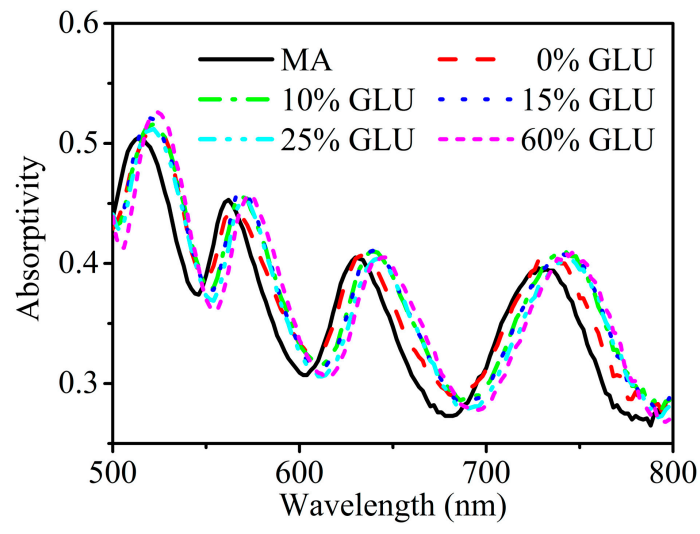

Figure 3. The effect on the absorption spectra for MA with drops of different concentrations of GLU on its surface. The black solid line shows the absorption spectrum of the MA.

After successively dropping 0,10,15, 25 and 60\% GLU solutions, the frequencies of the four absorption peaks all show slight red shifts, and the maximum red-shift values of the four absorption peaks are 12,12, 14 and $16 \mathrm{~nm}$, respectively. This fact indicates that the frequencies of the absorption 
peaks of the MA are sensitive to the variations of refractive index of GLU solutions. Therefore, the MA holds great promise for detecting refractive index changes of a sensing agent. A similar phenomenon has also been confirmed in a previous paper [28]. However, compared with the sensor in [28] which can only accomplish single-frequency responses, our present sensor can produce multiband responses to the refractive index changes. For the four absorption peaks, the average variations of refractive index $(\Delta \lambda / \Delta \mathrm{n})$ are about $113.2,113.2,132.1$, and $150.9 \mathrm{~nm} / \mathrm{RIU}$, which is of the same order as those of previously proposed sensors $[10,26]$. Meanwhile, the magnitudes of absorption peaks do not diminish evidently with the variations of refractive index.

The corresponding color change of the red shift effect mentioned above can be directly observed by the naked eyes. In practical terms, three kinds of GLU aqueous solutions with different concentrations $(0 \%, 20 \%$ and $60 \%)$ were selected to observe the color changes on the surface of the sample. In Figure $4 \mathrm{a}$, the center of the MA without any solution added is green. As the concentration of the aqueous GLU solution increases, instead of green, a magenta color gradually appears, as shown in Figure 4b-d. Finally, the center is dominated by magenta. The origin of this phenomenon lies in the red shift effects of absorption peaks as well as the color blending of light with different frequencies. Compared with traditional refractive index sensors, a small change of less than 0.05 in the liquid refractive index can be qualitatively distinguished just with the naked eyes. In the meanwhile, the detection process can be completed within a few minutes and the operation is very easy.

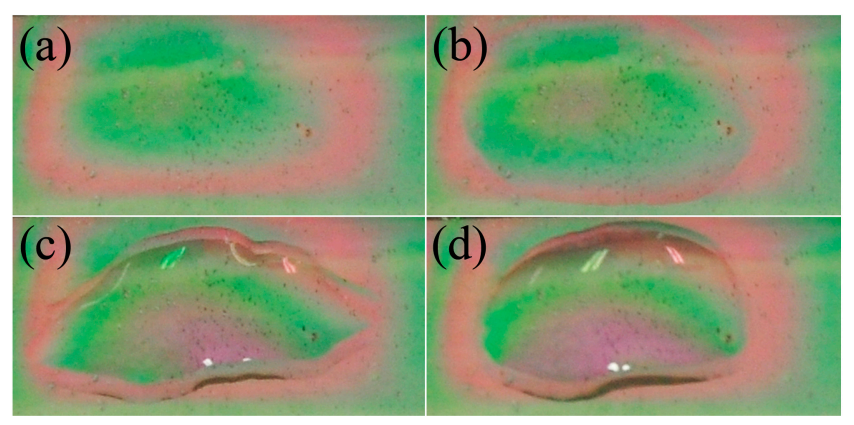

Figure 4. The photographs of the surface of MA with different concentrations of GLU (a) without any solution; (b) with UPW; (c) with 20\% GLU solution; (d) with 60\% GLU solution.

\section{Conclusions}

In conclusion, we have fabricated a MA with randomly distributed silver dendritic cells which exhibits multiband absorption in the visible light region for green, yellow and red light, respectively, via a "bottom-up" electrochemical deposition method. Incorporating a moderate gain material in the PVA dielectric layer is beneficial for the enhancement of absorption, and the absorption magnitude can be improved by about $30 \%$. In addition, the absorption peaks of MA are sensitive to variations of the refractive index of glucose solutions. It is shown that the corresponding color changes on the surface of the MA can be clearly observed by the naked eyes. Due to the aforementioned unique properties, the considered MA is extremely promising for applications in medical sensors, solar cells, and visible stealth technology. 


\section{Acknowledgments}

This work was supported by the National Nature Science Foundation of China under Grant No. 11174234, 51272215 and the National Key Scientific Program of China (under project No. 2012CB921503).

\section{Author Contributions}

X.P.Z. conceived the idea of fabricating the metamaterial sensor via the electrochemical deposition method. X.P.Z., H.L.M., and K.S. co-wrote the manuscript. H.L.M. and L.Z. fabricated the sample and completed all the experiments. All the authors reviewed the article.

\section{Conflicts of Interest}

The authors declare no conflict of interest.

\section{References}

1. Veselago, V.G. The electrodynamics of substances with simultaneously negative values of $\varepsilon$ and $\mu$. Sov. Phys. Usp. 1968, 10, 509-514.

2. Pendry, J.B. Negative refraction makes a perfect lens. Phys. Rev. Lett. 2000, 85, 3966.

3. Zhao, X.P. Bottom-up fabrication methods of optical metamaterials. J. Mater. Chem. 2012, 22, 9439-9449.

4. Schurig, D.; Mock, J.J.; Justice, B.J.; Cummer, S.A.; Pendry, J.B.; Starr, A.F.; Smith, D.R. Metamaterial electromagnetic cloak at microwave frequencies. Science 2006, 314, 977-980.

5. Tsakmakidis, K.L.; Boardman, A.D.; Hess, O. "Trapped rainbow" storage of light in metamaterials. Nature 2007, 450, 397-401.

6. Zhao, X.P.; Luo, W.; Huang, J.X.; Fu, Q.H.; Song, K.; Cheng, X.C.; Luo, C.R. Trapped rainbow effect in visible light left-handed heterostructures. Appl. Phys. Lett. 2009, 95, 07111.

7. Lee, J.-H.; Moon, H.-S.; Jang, I.-S.; Choi, J.-S.; Yook, J.-G.; Jung, H.-I. A planar split-ring resonator-based microwave biosensor for label-free detection of biomolecules. Sens. Actuators B Chem. 2012, 169, 26-31.

8. Ruffato, G.; Pasqualotto, E.; Sonato, A.; Zacco, G.; Silvestri, D.; Morpurgo, M.; Toni, A.D.; Romanato, F. Implementation and testing of a compact and high-resolution sensing device based on grating-coupled surface plasmon resonance with polarization modulation. Sens. Actuators $B$ Chem. 2013, 185, 179-187.

9. Landy, N.I.; Sajuyigbe, S.; Mock, J.J.; Smith, D.R.; Padilla, W.J. Perfect metamaterial absorber. Phys. Rev. Lett. 2008, 100, 207402.

10. Liu, N.; Mesch, M.; Weiss, T.; Hentschel, M.; Giessen, H. Infrared perfect absorber and its application as plasmonic sensor. Nano Lett. 2010, 10, 2342-2348. 
11. Cui, Y.X.; Fung, K.H.; Xu, J.; Ma, H.J.; Jin, Y.; He, S.L.; Fang, N.X. Ultrabroadband light absorption by a sawtooth anisotropic metamaterial slab. Nano Lett. 2012, 12, 1443-1447.

12. Kats, M.A.; Sharma, D.; Lin, J.; Genevet, P.; Blanchard, R.; Yang, Z.; Qazilbash, M.M.; Basov, D.N.; Ramanathan, S.; Capasso, F. Ultra-thin perfect absorber employing a tunable phase change material. Appl. Phys. Lett. 2012, 101, 221101.

13. Liu, X.M.; Zhao, Q.; Lan, C.W.; Zhou, J. Isotropic Mie resonance-based metamaterial perfect absorber. Appl. Phys. Lett. 2013, 103, 031910.

14. Liu, X.L.; Starr, T.; Starr, A.F.; Padilla, W.J. Infrared spatial and frequency selective metamaterial with near-unity absorbance. Phys. Rev. Lett. 2010, 104, 207403.

15. Ma, Y.; Chen, Q.; Grant, J.; Saha, S.C.; Khalid, A.; Cumming, D.R.S. A terahertz polarization insensitive dual band metamaterial absorber. Opt. Lett. 2011, 36, 945-947.

16. Grant, J.; Ma, Y.; Saha, S.; Lok, L.B.; Khalid, A.; Cumming, D.R.S. Polarization insensitive terahertz metamaterial absorber. Opt. Lett. 2011, 36, 1524-1526.

17. Shen, X.P.; Yang, Y.; Zang, Y.Z.; Gu, J.Q.; Han, J.G.; Zhang, W.L.; Cui, T.J. Triple-band terahertz metamaterial absorber: Design, experiment, and physical interpretation. Appl. Phys. Lett. 2012, $101,154102$.

18. Xiao, S.M.; Drachev, V.P.; Kildishev, A.V.; Ni, X.J.; Chettiar, U.K.; Yuan, H.K.; Shalaev, V.M. Loss-free and active optical negative-index metamaterials. Nature 2012, 466, 735-740.

19. Zhu, W.R.; Rukhlenko, I.D.; Premaratne, M. Light amplification in zero-index metamaterial with gain inserts. Appl. Phys. Lett. 2012, 101, 031907.

20. Fang, A.A.; Huang, Z.X.; Koschny, T.; Soukoulis, C.M. Overcoming the losses of a split ring resonator array with gain. Opt. Express 2011, 19, 12688-12699.

21. Liu, B.Q.; Zhao, X.P.; Zhu, W.R.; Luo, W.; Cheng, X.C. Multiple pass-band optical left-handed metamaterials based on random dendritic cells. Adv. Funct. Mater. 2008, 18, 3523-3528.

22. Zhu, W.R.; Zhao, X.P. Metamaterial absorber with random dendritic cells. EPJ. Appl. Phys. 2010, $50,21101$.

23. Zhu, W.R.; Zhao, X.P. Metamaterial absorber with dendritic cells at infrared frequencies. J. Opt. Soc. Am. B 2009, 26, 2382-2385.

24. Li, S.; Gong, B.Y.; Cao, D.; Pan, Z.Z.; Luo, C.R.; Zhao, X.P. A green-light gain-assisted metamaterial fabricated by self-assembled electrochemical deposition. Appl. Phys. Lett. 2013, 103, 181910.

25. Nunes, P.S.; Mortensen, N.A.; Kutter, J.P.; Mogensen, K.B. Refractive index sensor based on a 1D photonic crystal in a microfluidic channel. Sensors 2010, 10, 2348-2358.

26. Liu, Y.Z.; Salemink, H.W.M. All-optical on-chip sensor for high refractive index sensing in photonic crystals. Europhys. Lett. 2014, 107, 34008.

27. Song, Z.Y.; Feng, G.Y.; Zhang, T. Accurate measurement of the refractive index D-Glucose solution at various concentrations at different temperature. Chin. J. Lasers 2014, 41, 1208008.

28. Wang, X.N.; Luo, C.R.; Hong, G.; Zhao, X.P. Metamaterial optical refractive index sensor detected by the naked eye. Appl. Phys. Lett. 2013, 102, 091902.

(C) 2015 by the authors; licensee MDPI, Basel, Switzerland. This article is an open access article distributed under the terms and conditions of the Creative Commons Attribution license (http://creativecommons.org/licenses/by/4.0/). 